\title{
ESTUDOS SOBRE AS RELAÇÕES ENTRE ZINCO E FŌSFORO NA NUTRIÇÃO DA PLANTA *
}

\author{
0. E. Lopez G. \\ E. Malavolta $* * *$
}

\begin{abstract}
RESUMO
Uma série de experimentos com raízes destacadas e plantas inteiras de cevada (em solução nutritiva ou solo) deu apoio à conclusão de que a deficiência de zinco induzida pelo fosfato pode ser explicada pela operação de vários processos: inibição não competitiva de absorção do zinco; precipitação de fosfato de zinco na superfície das raízes; redução na translocação para a parte aérea; efeito de diluição resultante de alta velocidade de crescimento causada pelo fósforo no meio.
\end{abstract}

\section{INTRODUÇAO}

Muitos trabalhos mostraram que a deficiência de $\mathrm{Zn}$ está associada a altos níveis de $\mathrm{P}$ disponível no solo, ou que pode ser induzida pela aplicação de adubos fosfatados. Quando esta desordem nutricional pode ser prevenida ou corrigida pelo fornecimento de $\mathrm{Zn}$, o fenômeno é conhecido na literatura como "deficiência de $\mathrm{Zn}$ induzida pelo fosfato".

$O$ fato de que a presença de doses crescentes de $P$ afeta a disponibilidade de $\mathrm{Zn}$, podendo levar ao desenvolvimento de sintomas de deficiência ou de causar uma redução na concentração de $\mathrm{Zn}$ dos tecidos das plantas, tem sido verificado por numerosas pesquisas (BURLESON, DACUS e GERARD, 1961); LANGIN et al., 1962; BROWN e TIFFIN, 1962; WARD e al., 1963; ELLIS, DAVIS e THURLOW, 1964; JACKSON, HAY e MOORE, 1967; LO e REISENAUER, 1968; PAULSEN e ROTIMI, 1968; RUDGERDS et al., 1970; WARNOCK, 1970).

* Entregue para publicação em 27/12/1974.

Parte da tese de doutoramento apresentada à E. S. A. «Luiz de Queiroz», USP como parte das exigências do Curso Pós Graduado de Solos \& Nutrição de Plantas. Com ajuda da: FAPESP, BNDE, CNPq, CAPES, O. E. A., e USAID/BRASIL.

** Instituto Agronômico Nacional, Caacupé, Paraguay.

*** Departamento de Química e CENA, E. S. A. «Luiz de Queiroz», USP. 
Entretanto, os resultados obtidos por BOAWN, VIETS e GRAWFORD (1954) e BINGHAM (1963), não mostraram nenhum ef eito aparente do $P$ sobre a nutrição mineral do Zn. PAULI, ELLIS e MOSER (1968) verificaram, por outro lado, um ef eito positivo do fosfato na absorção de $\mathrm{Zn}$, mostrando as plantas de feijoeiro uma maior concentração do elemento nos seus tecidos.

A possibilidade de que o $\mathrm{P}$ parece bloquear a absorção de $\mathrm{Zn}$ e que a interação entre os dois nutrientes ocorre na superfície da raiz ou dentro delas, foi defendida por LANGIN et al., (1962).

STUKENHOLTZ et al., (1966) SHARMA et al., (1968 a, b) e PAULSEN e ROTIMI (1968) mostraram que o principal ef eito do fosfato foi uma inibição na translocação do $\mathrm{Zn}$ da raiz à parte aérea da planta. A esta conclusão chegaram após observar que a concentração de $\mathrm{Zn}$ no caule e folhas é mais agudamente afetada pela adubação fosfatada que a da raiz, órgão em que a concentração permanece quase inalterada.

Dada a informação nem sempre concordante encontrada na literatura foram formuladas as seguintes hipóteses de trabalho para explicar a influência do íon fosfato no aproveitamento do zinco pelas plantas:

a. diminuição na absorção por um efeito de inibição;

b. diminuição na absorção por precipitação do zinco na superfície da raiz;

c. diminuição na translocação da raiz para a parte aérea;

d. diminuição na disponibilidade do zinco no solo.

\section{MATERIAL E MÉTODOS}

\section{Absorção de $\mathrm{Zn}$ em presença de diversos fosfatos e cloretos em dife- rentes valores de $\mathrm{pH}$}

Os experimentos foram realizados com raízes destacadas de cevada (Hordeum vulgare var. breuns volla) de 7 dias de idade, seguindo a técnica descrita por EPSTEIN (1953).

As soluções de trabalho tinham as seguintes concentrações finais de $\mathrm{Zn}$ na forma $\mathrm{ZnCl}$, marcado com ${ }^{65} \mathrm{Zn}$ : $5 \times 10^{-6} \mathrm{M}, 5 \times 10^{-5} \mathrm{M}$ e $5 \times 10^{-4} \mathrm{M}$. Em cada uma dessas concentrações, a absorção de $\mathrm{Zn}$ foi estudada sob a influência das seguintes concentrações finais de fosfatos monobásicos de $\mathrm{Na}, \mathrm{K}, \mathrm{NH}_{4}$, Ca e $\mathrm{Mg}: 10^{-5} \mathrm{M}, 10^{-4} \mathrm{M}$ e $5 \times 10^{-3} \mathrm{M}$.

Os experimentos foram realizados nos $\mathrm{pH} 5,6$ e 7 ajustados com HCL ou $\mathrm{NaOH}$. Cada ensaio era repetido duas vezes.

$0{ }^{65} \mathrm{Zn}$ foi adicionado às soluções na forma de cloreto. A atividade específica era de 3 uCi por $\mathrm{mg}$ de $\mathrm{Zn}$. 
0 período de absorção era de 30 minutos, durante os quais as raízes permaneciam sob arejamento contínuo. A determinação da radioatividade das amostras era realizada introduzindo diretamente os tubos em um cintilador de cristal, tipo poço ligado a um conjunto registrador Hewlett-Packard modelo $5580 \mathrm{~B}$.

Os experimentos foram realizados com raízes destacadas de cevada de 7 dias de idade, seguindo uma modificação da metodologia do ítem anterior: no quarto dia a solução de sulfato de cálcio foi submetida pelas seguintes soluções de $\mathrm{Ca}\left(\mathrm{H}_{2} \mathrm{PO}_{4}\right)_{2}: 0,10^{-4} \mathrm{M}, 10^{-3} \mathrm{M}$ e $5 \times 10^{-3} \mathrm{M}$; cada uma destas soluções foi posta em um recipiente de dois litros de capacidade com um lote de plântulas de cevada.

\section{Absorção de $Z n$ em função da nutrição fosfałada prévia}

Os experimentos foram realizados com raízes destacadas de cevada de 7 dias de idade, seguindo uma modificação da metodologia do ítem anterior: no quarto dia a solução de sulfato de cálcio foi substituida pelas seguintes soluções de $\mathrm{Ca}\left(\mathrm{H}_{2} \mathrm{PO}_{4}\right)_{2}: 0,10^{-4} \mathrm{M}, 10^{-3} \mathrm{M}$ e $5 \times 10^{-3} \mathrm{M}$; cada uma destas soluções foi posta em um recipiente de dois litŕos de capacidade com um lote de plântulas de cevada.

\section{Ensaios com plantas inteiras}

Para estudar a translocação do $\mathrm{Zn}$ em função da nutrição fosfatada prévia, sementes de cevada foram postas a germinar em solução de $\mathrm{CaSO}_{4}$ ${ }^{10-4} \mathrm{M}$ no escuro e com arejamento contínuo, à semelhança do que se fez nos experimentos com raízes destacadas. No quarto dia o suporte com as plântulas foi lavado com água destilada e colocado novamente sobre recipientes de dois litros de capacidade contendo soluções com os seguintes tratamentos: $0,10^{-4} \mathrm{M}, 10^{-3} \mathrm{M}$ e $10^{-2} \mathrm{M}$ e $\mathrm{P}$ na forma de fosfato monossódico.

Inicialmente foi preparada a solução sem $P$ de HOAGLAND e ARNON (1950) diluida 1:10, à qual foram adicionadas soluções de reserva de fosfato monossódico a fim de se obter as concentrações finais desejadas.

As plantas permaneceram nessas soluções à luz durante três dias, em seguida as raízes eram lavadas e as plantas eram transferidas para as soluções contendo $\mathrm{Zn}$.

As soluções de trabalho contendo $\mathrm{Zn}$ foram também preparadas a partir da solução de HOAGLAND e ARNON (1950) sem P diluida. As concentrações finais de $\mathrm{Zn}$ foram as seguintes: $5 \times 10^{-6} \mathrm{M}, 5 \times 10^{-5} \mathrm{M}$ e $5 \times 10^{-4} \mathrm{M}$, com uma atividade específica de $3 \mathrm{uCi}$ por mg de $\mathrm{Zn}$.

De cada lote de plantas que receberam um pré tratamento com $P$ foram retiradas 30 plantas; destas 10 plantas foram colocadas em frasco contendo $200 \mathrm{ml}$ de cada uma das soluções contendo $\mathrm{Zn}$, onde permaneceram por mais três dias em arejamento contínuo. Após este período, as plantas foram retiradas dos frascos, as raízes lavadas com água e postas a secar entre 
folhas de papel toalha. As 10 plantas eram divididas em dois lotes de 5, separadas a raiz da parte aérea, introduzindo-se cada porção em um tubo de cultura para a determinação da sua radioatividade. Foi determinado o total de $\mathrm{Zn}$ absorvido e calculada a porcentagem do $\mathrm{Zn}$ encontrado na parte aérea.

$\mathrm{O}$ experimento foi realizado com as soluções de $\mathrm{Zn}$ ajustadas nos $\mathrm{pH}$ 5,6 e 7, sendo repetido duas vezes.

4. Absorção de Zn em função do tipo de solo, da calagem e da adubação fosfałada.

Foram utilizados três solos da região de Piracicaba, classificados pelo SERVIÇO NACIONAL DE PESQUISAS AGRONOMICAS (1960) como Regosol, (R) Latosol Vermelho Amarelo fase arenosa (LVa) e Latosol roxo (LR). bela 1 .

As características físicas e químicas dos solos se apresentam na Ta-

No ensaio em casa de vegetação empregou-se o delineamento experimental de blocos ao acaso, onde se compararam 6 tratamentos com três repetições.

Os tratamentos foram:

1. $\mathrm{Zn}+\mathrm{P}_{\mathbf{0}}$

2. $\mathrm{Zn}+\mathrm{P}_{1}$

3. $\mathrm{Zn}+\mathrm{P}_{2}$

4. $\mathrm{Zn}+\mathrm{P}_{0}+$ calagem

5. $\mathrm{Zn}+\mathrm{P}_{1}+$ calagem

6. $\mathrm{Zn}+\mathrm{P}_{2}+$ calagem

A quantidade de $\mathrm{Zn}$ aplicada a todos os tratamentos foi de $10 \mathrm{mg}$ de $\mathrm{Zn}$ por $\mathrm{kg}$ de solo, na forma de sulfato.

Os níveis de $\mathrm{P}$ foram de 0,200 e $400 \mathrm{mg}$ de $\mathrm{P}_{2} \mathrm{O}_{5}$ por $\mathrm{kg}$ de solo, fornecidos na forma de fosfato monossódico. 
Tabela 1 - Características físicas e químicas dos solos

\begin{tabular}{|c|c|c|c|c|}
\hline Análise mecânica & & $\underline{\mathbf{R}}$ & $\underline{L V a}$ & $\underline{\mathrm{LR}}$ \\
\hline Areia & $\%$ & 87,4 & 73,3 & 34,4 \\
\hline Limo & $\%$ & 1,8 & 12,1 & 24,1 \\
\hline Argila & $\%$ & 10,8 & 14,6 & 41,5 \\
\hline \multicolumn{5}{|l|}{ Análise química } \\
\hline $\mathrm{pH}$ & $\%$ & 5,6 & 5,0 & 5,9 \\
\hline C organico & $\%$ & 1,40 & 1,54 & 2,54 \\
\hline $\mathrm{PO}_{4}-3$ & $\mathrm{meq} / 100 \mathrm{~g}$ & 0,02 & 0,30 & 0,07 \\
\hline $\mathbf{K}^{+}$ & $\mathrm{meq} / 100 \mathrm{~g}$ & 0,04 & 0,08 & 0,48 \\
\hline $\mathrm{Ca}+2$ & $\mathrm{meq} / 100 \mathrm{~g}$ & 0,53 & 0,34 & 3,52 \\
\hline $\mathrm{Mg}^{+2}$ & $\mathrm{meq} / 100 \mathrm{~g}$ & 0,10 & 0,11 & 1,12 \\
\hline $\mathrm{H}^{+}$ & $\mathrm{meq} / 100 \mathrm{~g}$ & 3,58 & 4,69 & 3,97 \\
\hline $\mathrm{A} 1+3$ & $\mathrm{meq} / 100 \mathrm{~g}$ & 0,40 & 0,77 & 0,10 \\
\hline $\mathrm{Zn}^{+2}$ & $\mathrm{ppm}$ & 3,5 & 6,0 & 9,0 \\
\hline
\end{tabular}

A necessidade de cal dos solos foi determinado de acordo com CATANI e GALLO (1955), aplicando-se as quantidades de carbonato de cálcio, p. a. em suspensão, de maneira a atingir o pH 6,5. Os solos foram deixados incubados durante 20 dias com uma umidade aproximada de 60 a $70 \%$ da capacidade de campo.

Para evitar uma possível interferência de qualquer elemento essencial não sujeito ao estudo, aplicou-se aos solos uma adubação complementar, em solução dos seguintes nutrientes: $\mathrm{N}, 200 \mathrm{ppm} ; \mathrm{K}_{2} \mathrm{O}, 200 \mathrm{ppm} ; \mathrm{Mg}$ 34 ppm; S, 124 ppm; B, 1,2 ppm; Cu, 2,5 ppm; Fe, 5 ppm; Mn, 1,4 ppm; Mo, 0,5 ppm.

A atividade específica da solução de $\mathrm{Zn}$ aplicada foi de $5 \mathrm{uCi}$ por $\mathrm{mg}$ de $\mathrm{Zn}$. A atividade total aplicada em cada tratamento foi de $50 \mathrm{uCi}$ por vaso.

Como planta indicadora empregou-se a cevada, na proporção de 15 sementes por vaso, tendo-se procedido ao desbaste 8 dias após a germinação para manter 8 plantas por vaso.

A colheita da parte aérea da planta foi realizada aos 45 dias. 0 material colhido foi seco em estufa a $60-70^{\circ} \mathrm{C}$ durante 72 horas, após o que procedeu-se a pesagem e moagem do material.

A radioatividade das amostras foi determinada colocando-se $0,5 \mathrm{~g}$ de material moido em tubos de cultura e introduzindo-o no cintilador de cristal, tipo poço, ligado ao conjunto Hewlett-Packard, modelo $5580 \mathrm{~B}$.

$\mathrm{A}$ determinação de $\mathrm{P}$ e $\mathrm{Zn}$ foi realizada no extrato nítrico-perclórico do mesmo material cuja atividade foi medida. O P foi analisado colorime- 
tricamente pelo método fosfovanodomilibdico (MALAVOLTA 1964) e o $\mathrm{Zn}$ no espectrofotômetro de absorção atômica EEL, modelo 140 .

Foram calculados o valor $\mathrm{L}$ dos solos e a quantidade total de $\mathrm{Zn}$ na parte aérea das plantas. $O$ efeito dos diferentes tratamentos no crescimento e na concentração de $\mathrm{P}$ e $\mathrm{Zn}$ da planta foi analizada estatisticamente de acordo com PIMENTEL GOMES (1970).

\section{RESULTADOS E DISCUSSAOO}

1. Influência de fosfatos e cloretos na absorção do $\mathrm{Zn}$ em vários valores de $\mathrm{pH}$

A Tabela 2 mostra que, independentemente da fonte de $P$ a absorção de $\mathrm{Zn}$ diminuiu quando a concentração do primeiro era elevada de $10^{-5} \mathrm{M}$ a $5 \times 10^{-3} \mathrm{M}$. A diminuição ocorre em todos os valores de $\mathrm{pH}$ e níveis de $\mathrm{Zn}$. 


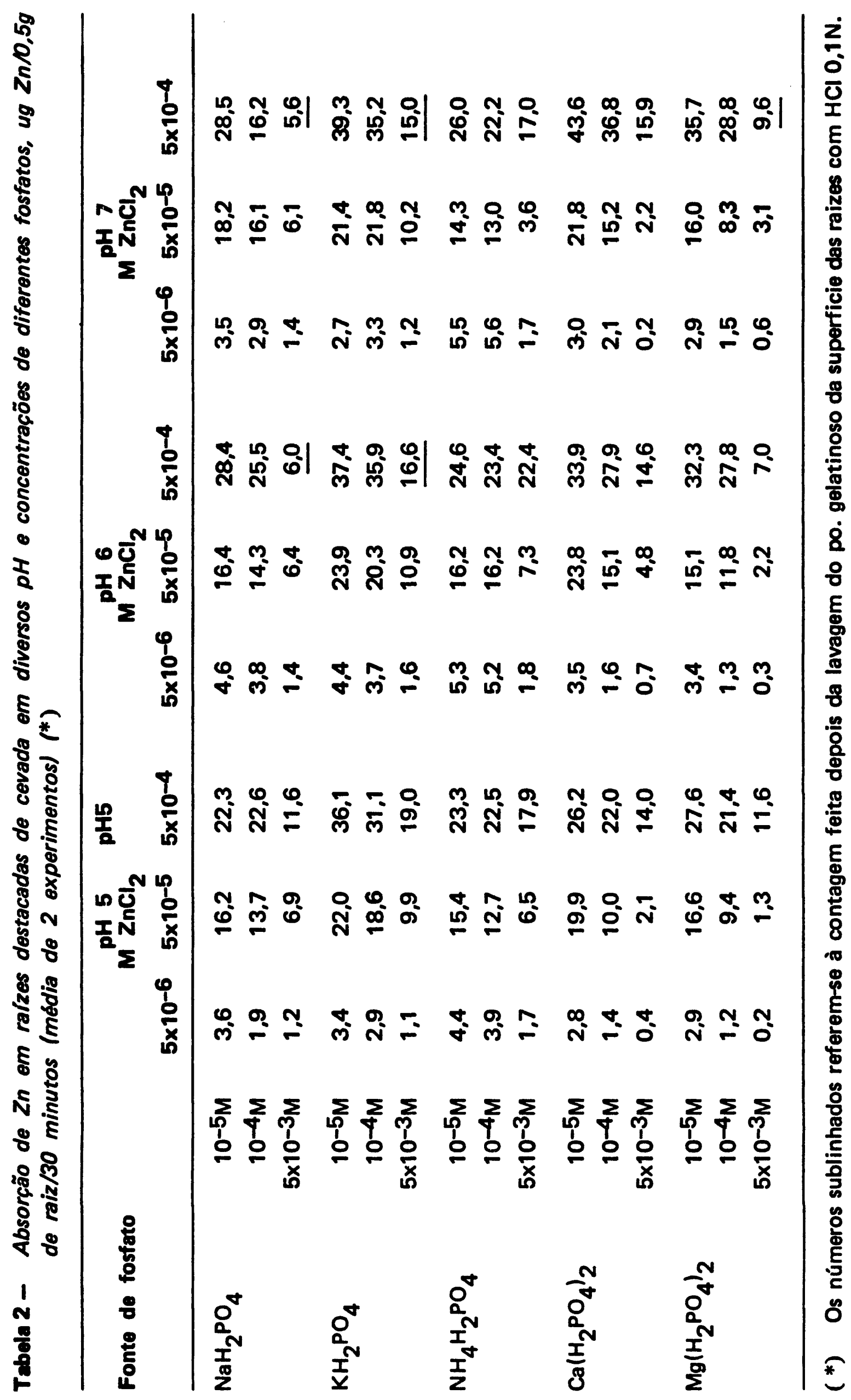


Um efeito diferente do iônio acompanhante é observado particularmente no nível mais baixo de $\mathrm{Zn}$. Os fosfatos da $\mathrm{Ca}$ e $\mathrm{Mg}$ reduziram a absorção mais acentuadamente que as demais. 0 iônio amoniacal mostrou a menor influência. Que o fosfato de Ca tinha o maior efeito na absorção do $\mathrm{Zn}$ foi verificado também por BINGHAN \& GARBER (1960) quer, por outro lado, verificaram a pequena influência do $\mathrm{NH}_{4}{ }^{+}$.

Os resultados não mostram uma variação consistente na absorção sob o efeito do $\mathrm{pH}$ o que está de acordo com os resultados obtidos por HAAG \& SARRUGE (1965), BOWEN (1969) e CHAUDRY \& LONERAGAN (1972-a) trabalhando na faixa 5,0 a 7,0 .

Os experimentos em que foram usados cloretos em lugar de fosfatos foram planejados para verificar se a diminuição na absorção do $\mathrm{Zn}$ era devida ao catiônio acompanhante ou ao próprio aniônio. Os resultados obtidos (Tabela 3) mostram que a absorção diminuiu quando aumenta a concentração de cloreto. $\mathrm{O}$ efeito diferente do iônio acompanhante pode também ser observado. A influência de $\mathrm{Ca}^{+2}$ e $\mathrm{Mg}^{+2}$ é geralmente maior que a dos catiônios monovalentes. Usando nitratos CHAUDRY \& LONERAGAN (1972-b) acharam que o efeito inibitório era mais acentuado na presença de $\mathrm{NH}_{4}+$ do que na de $\mathrm{Na}^{+}$, o $\mathrm{K}^{+}$ocupando em lugar intermediário. SCHMLD et al., (1965) observaram também forte efeito despressivo do $\mathrm{Ca}^{+2}$ enquanto $\mathrm{BO}$ WEN (1969), por sua vez, não encontrou efeito marcante dos catiônios alcalinos no processo. A Tabela 3 mostra também que a absorção foi menor em $\mathrm{pH}$ 6,0 e 7,0, o que não ficou claro no ensaio anterior. 


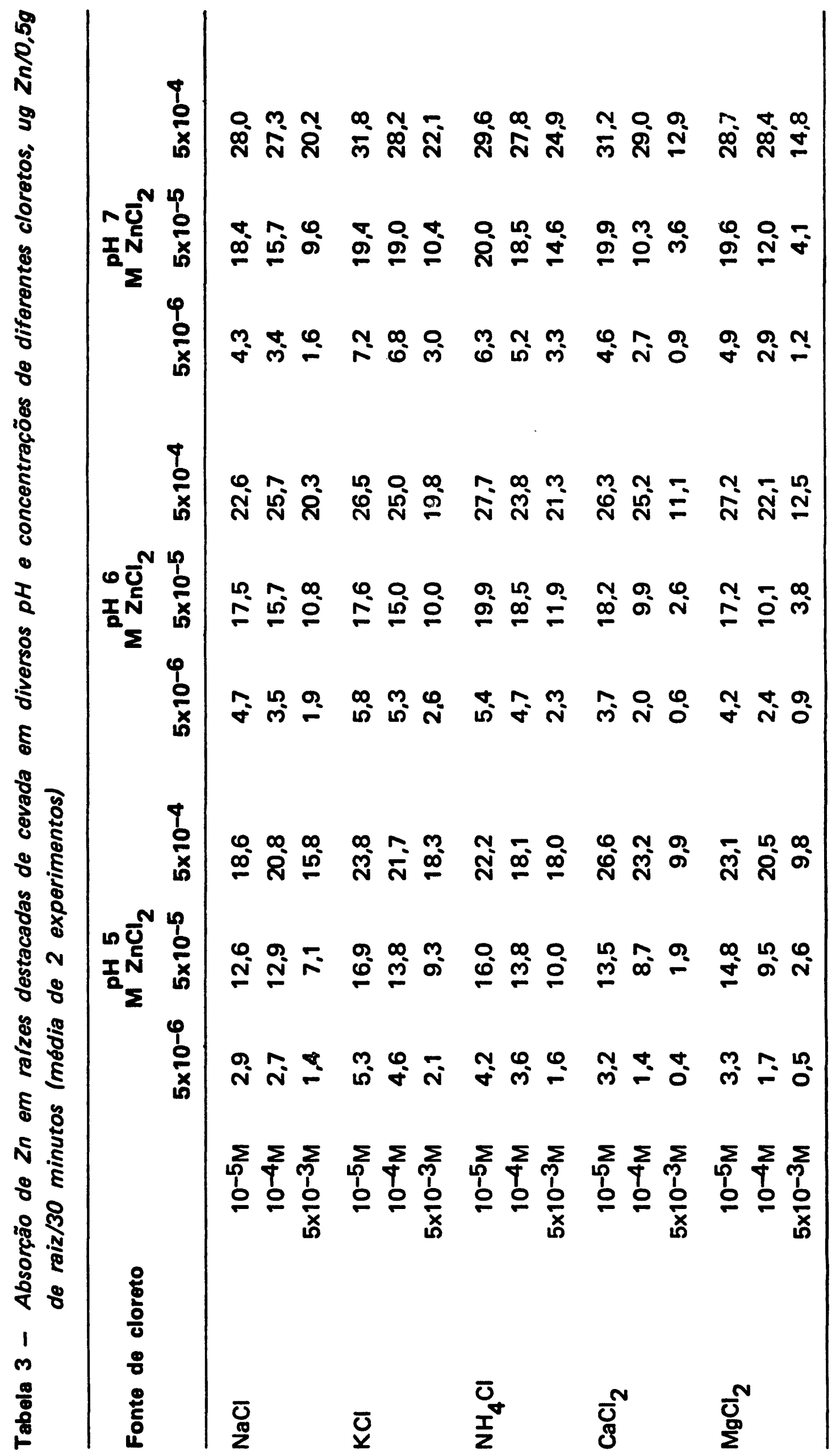


A conhecida transformação usada em cinética enzimática foi aplicada aos dados com o fim de obter informação relativa à natureza do efeito inibitório. Os valores da ordenada do ponto de inter secção (1/V) e do coeficiente angular $(\mathrm{Km} / \mathrm{V})$ são dados na Tabela 4 . Torna-se claro que a inibição é do tipo não competitivo e que a natureza do efeito não depende do $\mathrm{pH}$. Como se viu previamente a influência inibitória dos catiônios divalentes é mais forte que a dos monovalentes. A influência dos fosfatos é maior que a dos cloretos. $\mathrm{E}$ lícito concluir, pois, que o efeito observado com os diferentes fosfatos se desdobra em duas componentes: o do fosfato e aquele do iônio acompanhante.

\section{Influência da nutrição fosfatada prévia na absorção do zinco}

Como demonstra a Tabela 5 , a absorção do $\mathrm{Zn}$ foi maior no caso das plantas que haviam recebido previamente $\mathrm{P}$ e aqui também ela cresce com o aumento da concentração do micronutriente no meio. Esses resultados parecem indicar que o estado nutricional da planta com respeito ao $P$ não é a causa do efeito inibitório o que dá apoio ao ponto de vista apresentado por GANIRON et al., (1969).

Tabela 5 - Absorção do $\mathrm{Zn}$ (ug $\mathrm{Zn} / 0,5 \mathrm{~g}$ raízes/30 minutos) por raizes destacadas sob influência da nutrição fosfatada prévia

\begin{tabular}{|c|c|c|c|c|c|c|c|c|c|c|c|c|}
\hline \multirow[t]{2}{*}{$\mathrm{ZnC1}_{2}$} & \multicolumn{3}{|c|}{$\begin{array}{l}\mathrm{OM} \\
\mathrm{pH}\end{array}$} & \multicolumn{3}{|c|}{$\begin{array}{l}\mathrm{CaH}_{2}\left(\mathrm{PO}_{4}\right)_{2} \\
10-{ }^{4} \mathrm{M} \\
\mathrm{pH}\end{array}$} & \multicolumn{3}{|c|}{$\begin{array}{c}10-{ }^{-3} \mathrm{M} \\
\mathrm{pH}\end{array}$} & \multicolumn{3}{|c|}{$\underset{\mathrm{pH}}{5 \times 10-3 \mathrm{M}}$} \\
\hline & 5,0 & 6,0 & 7,0 & 5,0 & 6,0 & 7,0 & 5,0 & 6,0 & 7,0 & 5,0 & 6,0 & 7,0 \\
\hline $5 \times 10^{-6} \mathrm{M}$ & 9 & 10 & 10 & 10 & 10 & 11 & 11 & 11 & 12 & 9 & 9 & \\
\hline $5 \times 10^{-5} \mathrm{M}$ & 21 & 25 & 27 & 25 & 30 & 35 & 28 & 32 & 34 & 26 & 30 & 31 \\
\hline $5 \times 10^{-}{ }^{4} \mathrm{M}$ & 31 & 35 & 39 & 37 & 39 & 41 & 38 & 45 & 47 & 39 & 45 & 46 \\
\hline
\end{tabular}


Tabela 4 - Intercepção e inclinação da reta das reciprocas das velocidades de absorção de $\mathrm{Zn}$ - das concentrą̧ōes de $\mathrm{ZnCl}_{2}$

\begin{tabular}{|c|c|c|c|c|c|c|c|}
\hline & $\begin{array}{l}\text { Concentracão } \\
\text { do substrato }\end{array}$ & $\begin{array}{l}\mathrm{pH} \\
1 / \mathrm{V}\end{array}$ & $\mathrm{Km} / \mathbf{V}$ & $\begin{array}{l}\mathrm{PH} \\
\mathbf{1} / \mathrm{V}\end{array}$ & $\mathrm{Km} / \mathrm{V}$ & $\begin{array}{l}\mathrm{pH} \\
\mathbf{1 N}\end{array}$ & $\mathrm{Km} / \mathrm{V}$ \\
\hline \multirow{4}{*}{$\mathrm{NaH}_{2} \mathrm{PO}_{4}$} & & $\times 10^{-2}$ & $\times 10^{-5}$ & $\times 10^{-2}$ & $\times 10^{-5}$ & $\times 10^{-2}$ & $\times 10^{-5}$ \\
\hline & $10^{-5} 5_{M}$ & 4,8 & 0,11 & 4,5 & 0,08 & 3,7 & 0,12 \\
\hline & $10^{-4} M$ & 3,8 & 0,24 & 4,9 & 0,10 & 5,2 & 0,14 \\
\hline & $5 \times 10^{-3} M$ & 7,9 & 0,37 & 13,7 & 0,28 & 14,3 & 0,28 \\
\hline \multirow[t]{3}{*}{$\mathrm{NaCl}$} & $10^{-5} M$ & 5,4 & 0,14 & 4,4 & 0,08 & 4,0 & 0,09 \\
\hline & $10^{-4} M$ & 4,7 & 0,16 & 4,2 & 0,12 & 4,2 & 0,12 \\
\hline & $5 \times 10^{-3} M$ & 6,8 & 0,32 & 4,6 & 0,24 & 5,1 & 0,28 \\
\hline \multirow{3}{*}{$\mathrm{KH}_{2} \mathrm{PO}_{4}$} & $10-5 M$ & 2,5 & 0,13 & 2,6 & 0,10 & 2,2 & 0,17 \\
\hline & $10^{-4} M$ & 3,1 & 0,15 & 2,7 & 0,12 & 2,8 & 0,13 \\
\hline & $5 \times 10^{-3} M$ & 3,6 & 0,43 & 5,2 & 0,28 & 4,4 & 0,39 \\
\hline \multirow[t]{3}{*}{$\mathrm{KCL}$} & $10^{-5} \mathrm{M}$ & 4,5 & 0,07 & 4,4 & 0,06 & 3,7 & 0,05 \\
\hline & $10^{-4} M$ & 5,3 & 0,08 & 4,7 & 0,07 & 4,2 & 0,05 \\
\hline & $5 \times 10^{-3} M$ & 5,9 & 0,21 & 5,9 & 0,16 & 6,1 & 0,13 \\
\hline \multirow{3}{*}{$\mathrm{NH}_{4} \mathrm{H}_{2} \mathrm{PO}_{4}$} & $10^{-5} M$ & 4,6 & 0,09 & 4,6 & 0,07 & 5,2 & 0,06 \\
\hline & $10^{-4} M$ & 5,4 & 0,10 & 4,7 & 0,07 & 5,6 & 0,06 \\
\hline & $5 \times 10^{-3} M$ & 8,2 & 0,25 & 7,0 & 0,24 & 14,6 & 0,22 \\
\hline \multirow{3}{*}{$\mathrm{NH}_{4} \mathrm{Cl}$} & $10-5_{M}$ & 4,9 & 0,09 & 4,0 & 0,07 & 3,7 & 0,06 \\
\hline & $10^{-4} M$ & 5,4 & 0,11 & 4,3 & 0,08 & 4,2 & 0,07 \\
\hline & $5 \times 10^{-3} M$ & 4,7 & 0,29 & 4,6 & 0,19 & 4,0 & 0,13 \\
\hline \multirow{3}{*}{$\mathrm{Ca}\left(\mathrm{H}_{2} \mathrm{PO}_{4}\right)_{2}$} & $10^{-5} M$ & 3,1 & 0,16 & 2,3 & 0,13 & 2,2 & 0,15 \\
\hline & $10^{-4} M$ & 4,1 & 0,33 & 2,0 & 0,30 & 2,8 & 0,22 \\
\hline & $5 \times 10^{-3} M$ & 14,2 & 0,18 & 6,5 & 0,68 & 1,2 & 2,50 \\
\hline \multirow{3}{*}{$\mathrm{CaCl}_{2}$} & $10^{-5} \mathrm{M}$ & 4,4 & 0,13 & 4,0 & 0,11 & 3,4 & 0,09 \\
\hline & $10^{-4} M$ & 4,5 & 0,33 & 5,1 & 0,22 & 4,9 & 0,16 \\
\hline & $5 \times 10^{-3} M$ & 18,4 & 1,16 & 15,2 & 0,76 & 12,6 & 0,49 \\
\hline \multirow{3}{*}{$\mathrm{Mg}\left(\mathrm{H}_{2} \mathrm{PO}_{4}\right)_{2}$} & $10^{-5} M$ & 3,4 & 0,15 & 4,0 & 0,12 & 3,3 & 0,15 \\
\hline & $10^{-4} M$ & 3,9 & 0,39 & 2,3 & 0,37 & 4,6 & 0,31 \\
\hline & $5 \times 10^{-3} M$ & 16,1 & 2,42 & 12,5 & 1,60 & 12,8 & 0,77 \\
\hline \multirow{4}{*}{$\mathrm{MgCl}_{2}$} & $10^{-5} M$ & 4,8 & 0,12 & 3,7 & 0,10 & 3,6 & 0,08 \\
\hline & $10^{-4} M$ & 4,8 & 0,27 & 5,5 & 0,18 & 4,9 & 0,14 \\
\hline & $5 \times 10^{-3} M$ & 14,1 & 0,93 & 11,4 & 0,50 & 11,4 & 0,36 \\
\hline & 0 & 3,0 & 0,05 & 3,5 & 0,04 & 3,3 & 0,04 \\
\hline
\end{tabular}




\section{Influência da nutrição fosfatada prévia na translocação do $\mathrm{Zn}$}

0 "índice de translocação" definido por RUSSEL \& MARTIN (1953) como a \% do elemento encontrado no topo relativamente ao total da planta foi usado para preparar a Tabela 6 a qual mostra que o fosfato previamente absorvido diminuiu a quantidade de $\mathrm{Zn}$ translocada particularmente em $\mathrm{pH} 6,0$ e $\mathrm{pH} 7,0$. Por esse processo, portanto, o teor de $\mathrm{Zn}$ no topo pode ser ainda mais diminuido, uma situação já conhecida para o ferro.

Tabela 6 - Translocação do $\mathrm{Zn}$ (tôpo \% do total absorvido) em «seedlings» intactos sob influência da nutrição fosfatada prevvia.

\begin{tabular}{|c|c|c|c|c|c|c|c|c|c|c|c|c|}
\hline \multirow[t]{2}{*}{$\mathrm{ZnCl}_{2}$} & \multicolumn{3}{|c|}{$\begin{array}{l}\mathrm{OM} \\
\mathrm{pH}\end{array}$} & \multicolumn{3}{|c|}{$\begin{array}{c}\mathrm{NaH}_{2} \mathrm{PO}_{4} \\
10-{ }_{4} \mathrm{M} \\
\mathrm{pH}\end{array}$} & \multicolumn{3}{|c|}{$\begin{array}{c}10-{ }^{-3} \mathrm{M} \\
\mathrm{pH}\end{array}$} & \multicolumn{3}{|c|}{$\begin{array}{c}10-{ }^{2} \mathrm{M} \\
\mathrm{pH}\end{array}$} \\
\hline & 5,0 & 6,0 & 7,0 & 5,0 & 6,0 & 7,0 & 5,0 & 6,0 & 7,0 & 5,0 & 6,0 & 7,0 \\
\hline $5 \times 10^{-6} \mathrm{M}$ & 27 & 28 & 30 & 27 & 33 & 26 & 26 & 30 & 25 & 22 & 21 & 25 \\
\hline $5 \times 10^{-5} \mathrm{M}$ & 17 & 25 & 29 & 20 & 15 & 21 & 19 & 10 & 10 & 16 & 11 & 20 \\
\hline $5 \times 10^{-4} \mathrm{M}$ & 27 & 31 & 33 & 33 & 35 & 26 & 26 & 30 & 26 & 25 & 26 & 26 \\
\hline
\end{tabular}

4. Influência do tipo do solo, calagem e dose de $\mathbf{P}$ na absorção do $\mathbf{Z n}$.

Os resultados resumidos na Tabela 6 mostram que a produção de matéria seca e o teor de $\mathrm{P}$ usualmente aumentaram com as aplicações de $\mathrm{P}$ e de calcário enquanto o teor de $\mathrm{Zn}$ no topo das plantas diminuiu, o que pode ser explicado por um efeito diluição na maioria dos casos. De fato, uma correlação negativa foi achada entre $\mathrm{P}$ aplicado e teor de $\mathrm{Zn}\left(\mathrm{r}=0,70^{* *}\right)$ e entre os teores dos dois elementos $\left(r=067^{* *}\right)$. Por outro lado, os valores " $L$ " mostraram uma certa tendência para aumentar com a dose de $\mathrm{P}$ aplicada; isto sugere que não houve redução na disponibilidade do $\mathrm{Zn}$, resultado confirmado por determinações do Zn disponível usando soluções extrătoras; esses resultados confirmam os de MARINHO \& IGUE (1972). 
Tabela 7 - Efeito do tipo de solo, calagem e dose de $P$ na produção de matéria seca, teores de $\mathrm{Zn}$ e $\mathrm{P}$ no topo e no valor «L».

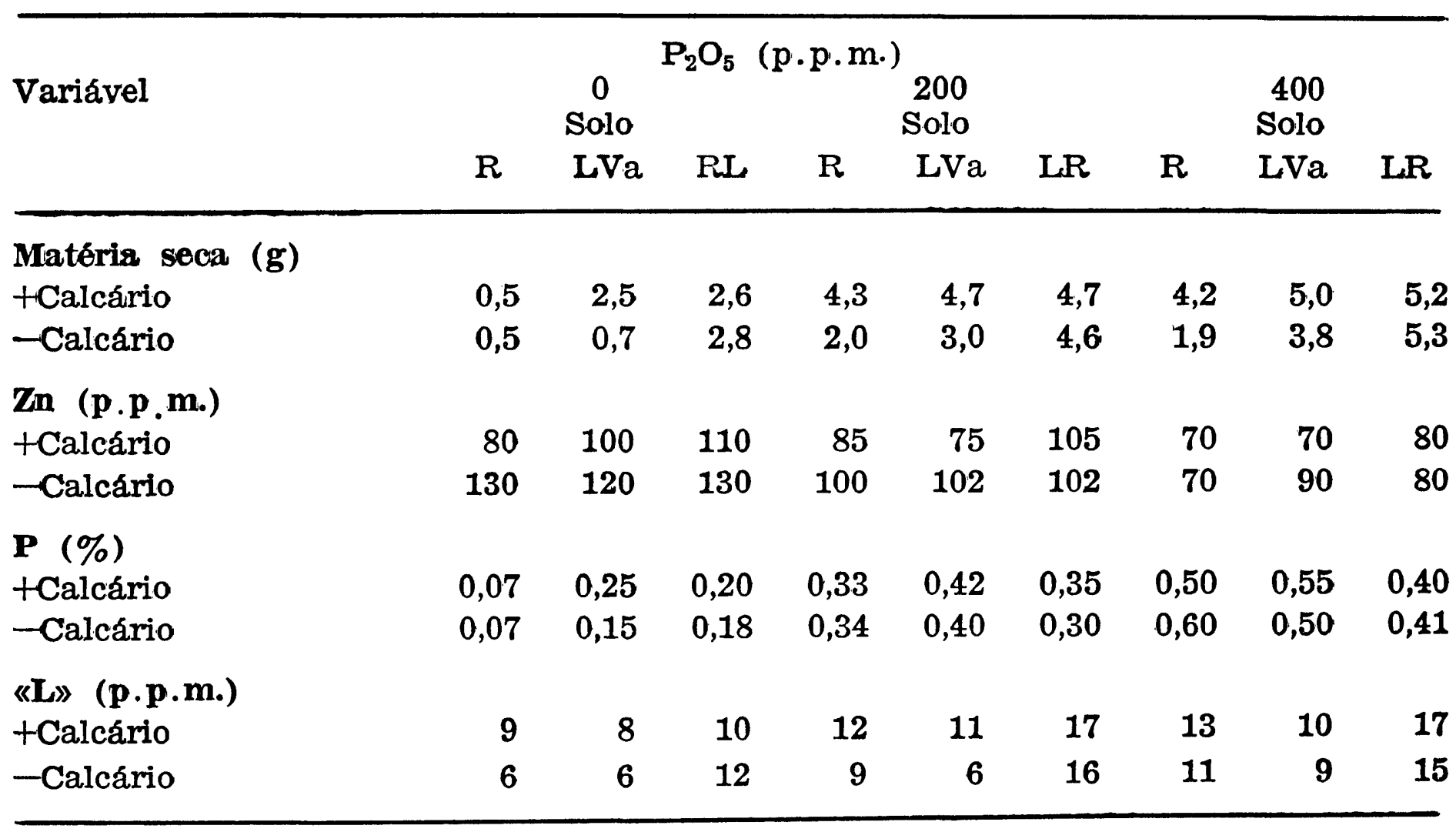

\section{CONCLUSOES}

Com a finalidade de estudar a influência do ín fosfatado no aproveitamento do zinco pelas plantas, foram realizados ensaios com raízes destacadas, plantas inteiras e com solos em casa de vegetação, usando a cevada (Hodeum vulgare L. var. breuns volla) como plantas indicadora.

Os ensaios com raízes destacadas e com plantas inteiras foram feitos em três concentrações de $\mathrm{Zn}\left(5 \times 10^{-6} \mathrm{M}, 5 \times 10^{-5} \mathrm{M}\right.$ e $\left.5 \times 10^{-4} \mathrm{M}\right)$ e nos $\mathrm{pH} 5,6$ e 7 .

A absorção de $\mathrm{Zn}$ por raízes destacadas de 7 dias de idade foi estudada com $0,5 \mathrm{~g}$ de raiz no tempo de 30 minutos, em presença das concentrações $10^{-5} \mathrm{M}, 10^{-4} \mathrm{M}$ e $5 \times 10^{-3} \mathrm{M}$ de $\mathrm{Na}, \mathrm{K}, \mathrm{NH}_{4}, \mathrm{Ca}$ e $\mathrm{Mg}$, nas formas de fosfato e cloreto.

A absorção de $\mathrm{Zn}$ foi também estudada com raízes destacadas de plantulas que permaneceram crescendo previamente em soluções de fosfato de concentração crescente na forma monocálcica.

Com plantas inteiras foi estudada a translocação de $\mathrm{Zn}$ em função da nutrição fosfatada prévia, usando-se as seguintes concentrações de P: $0,10^{-4} \mathrm{M}, 10^{-3} \mathrm{M}$ e $10^{-2} \mathrm{M}$.

0 efeito da adubação fosfatada e da calagem no aproveitamento do Zn foi estudado em três solos: Regosol, Latosol vermelho amarelo fase arenosa e Latosol roxo. Os níveis de $P^{2} O^{5}$ aplicados aos solos foram: 0, 200 
e $400 \mathrm{ppm}$. A calagem foi feita visando os solos atingirem o pH 6,5. Em todos os tratamentos foi aplicado $10 \mathrm{mg}$ de $\mathrm{Zn}$ por $\mathrm{kg}$ de solo. No ensaio empregaram-se $1000 \mathrm{~g}$ de solo por vaso, que receberam uma adubação complementar de todos os nutrientes essenciais. 0 ensaio foi colhido aos 45 dias.

Em todos os experimentos foi usado ${ }^{65} \mathrm{ZN}$ como traçador.

As conclusões foram as seguintes:

a. Todos os sais causaram uma inibição não competitiva na absorção de $\mathrm{Zn}$.

b. A natureza do efeito não depende do $\mathrm{pH}$.

c. A inibição é maior quando o fosfato ou cloreto estão acompanhados dos cátions divalentes.

d. 0 efeito do fosfato foi maior que do cloreto na inibição da adsorção de $\mathrm{Zn}$.

e. $O$ estado nutricional prévio das plantas em fosfato não diminui a adsorção de $\mathrm{Zn}$, tendo pequeno efeito depressivo na translocação quando se usarem baixas concentrações de $\mathrm{Zn}$ e altas de $\mathrm{P}$.

f. A adubação fosfatada e a calagem diminuem a concentração de $\mathrm{Zn}$, na planta, não influindo na disponibilidade de $\mathrm{Zn}$ no solo, avaliada por métodos químicos e biológicos.

\section{SUMMARY}

STUDIES ON THE ZINC PHOSPHATE RELATIONSHIPS IN PLANT NUTRITION

This paper deals with the results of experiments designed to study the nature of the effect of phosphate $(\mathrm{P})$ ion on the absorption of zinc $(\mathrm{Zn})$ by plants. Due to the conflicting results found in the literature the following working hypothesis were raised and checked:

a. $\mathrm{P}$ decreases $\mathrm{Zn}$ levels in the top by interfering with its uptake at the root level;

b. decreases $\mathrm{Zn}$ uptake by roots due to preciptation at their surface;

c. $\mathrm{P}$ decreases $\mathrm{Zn}$ content in the top by a negative effect on the upward movement of the absorbed element;

d. $\mathrm{P}$ decreases $\mathrm{Zn}$ uptake in soil conditions by rendering it unavailable.

Experiments with excised roots and whole plants were conducted using barley as an indicator.

Experiments with excised roots and whole plants were carried out at there $\mathrm{Zn}$ concentraions $\left(5 \times 10^{-6} \mathrm{M}, 5 \times 10^{-5} \mathrm{M}\right.$, and $\left.5 \times 10^{-4} \mathrm{M}\right)$ a $\mathrm{pH} 5.0$ 
6.0 and 7.0. In the former trials $0,5 \mathrm{~g}$ of roots excised from 7 day old seedlings were used during an uptake period of 30 minutes; phosphates of $\mathrm{Na}$, $\mathrm{K}, \mathrm{NH}_{4}, \mathrm{Ca}$ and $\mathrm{Mg}$ were used at the concentrations of $10^{-5} \mathrm{M}, 10^{-4} \mathrm{M}$ and $5 \times 10^{-3} \mathrm{M}$. In order to better define a possible role of the accompanying cation same experiments were repeated replacing the phosphate by the chloride ion.

The effect of the internal $P$ status of the plant both of he upake and translocation was studied using respectively roots excised from plants previously grown in phosphate solutions $\left(10^{-4} \mathrm{M}, 10^{-3} \mathrm{M}\right.$, and $\left.5 \times 10^{-3} \mathrm{M}\right)$ and whole plants $\left(0,10^{-4} \mathrm{M}, 10^{-3} \mathrm{M}\right.$ and $\left.10^{-2} \mathrm{M}\right)$.

The influence of phosphatic fertilization was assessed by supplying varying rates of $P(0,200$ and $400 \mathrm{ppm})$ plus other macro and micronutrients in standard amounts to three soil types (Regosol, Reed yellow Latosol sand phase and Reed Latosol) in the presence and in the abscence of lime.

In all experiments ${ }^{65} \mathrm{Zn}$ was used as tracer.

The main conclusions were the following:

a. all salts used in soilless experiments reduced $\mathrm{Zn}$ uptake by non competitive inhibition;

b. the size of the effect is $\mathrm{pH}$ dependent;

c. inhibition is greater when either $\mathrm{P}$ or $\mathrm{Cl}$ are accompanied divalent cation;

d. effect is higher than Cl's;

e. Precipitation of $\mathrm{Zn}$ by $\mathrm{P}$ in soilltss mtdium may take place depending upon concentration and $\mathrm{pH}$, the phenomenon occurring either in them edium it self or at the surface;

f. previous phosphatic nutrition did not decrease $\mathrm{Zn}$ uptake having however, slight, negative effect on translocation when high levels of the anion were used in the pretreament combined with low concentrations of the micronutrient during the experimental period;

g. phosphatic fertilization and liming decreased $\mathrm{Zn}$ corcentration in the olant having no lowering effect on its availability in the soil as revealed by a chemical method, the first variable actually increasing absorptionas determined by the " $L$ " values thereof.

\section{LITERATURA CITADA}

BINGHAM, F. T. (1963) - Relation between phosphorus and micronutrients in plants. Soil Sci. Amer. Proc. 27 : 389-391.

BINGHAM, F. T. \& GARBER, M. J. (1960) - Solubility and availability of micronutrients in relation to phosphorus fertilization. Soil Sci. Soc. Amer. Proc. 24 : 209-213. 
BOAWN, L. C., VIETS, F. S. \& CRAWFORD, C. L. (1954) - Effect of phosphate fertilizers on zinc nutrition of field beans. Soil. Sci., 78:1-7.

BOWEN, J. E. (1969) - Absorption of copper, zinc, and manganese by sugarcane leaf tissue. Plant. Physiol. $44: 255-261$.

BROWN, J. C. \& TIFFIN, L. O. (1962) - Zinc deficiency and iron chlorosis dependent on the plant species and nutrient-element balance in Tulare clay. Agronomy J., 54 : 356-358.

BURLESON, C. A., DACUS, A. D. \& GERARD, C. J. (1961) - The effect of phosphorus fertilization on the zinc nutrition of several irrigated crops. Soil Sci. Soc. Amer. Proc. 25 : 365-368.

CATANI, R. A. \& GALLO, J. R. (1955) - Avaliação da exigência em calcário dos solos do Estado de São Paulo, mediante correlıção entre pH e a porcentagem de saturação em bases. Rev. Agric. Piracicaba, 30 : 49-60.

CHAUDHRY, F. M. \& LONERAGAN, J. F. (1972a) - Zinc absorption by wheat seedlings: $I$. Inhibition by macronutrients ions in short-term experiments and its relevance to long-term zinc nutrition. Soil Sci. Soc. Amer. Proc. 36 : 323-327.

CHAUDHRY, F. M. \& LONERAGAN, J. F. (1972b) - Zinc absorption by wheat seedlings III. Inhibition by hydrogen ions and by micronutrient cations. Soil Sci. Soc. Amer. Proc. 36 : 327-331.

ELLIS, R., DAVIS, J. F. \& THURLOW, D. L. (1964) - Zinc availability in calcareous Michigan soils as influenced by phosphorus level and temperature. Soil Sci Soc. Amer. Proc. 28 : 83-86.

FPSTEIN, E. (1953) - Ion absorption by plant. roots Proc. $4^{\text {th }}$ Annual Oak Ridge Siummer Symposium. U. S. Atomic Energy Comm.: 418-434.

GANIRON, R. B., ADRIANO, D. C., PAULSEN, G. M. \& MURPHY, L. S. (1969) Effect of phosphorus carriers and zing sources on phosphorus-zinc interaction in corn. Soil Sci. Amer. Proc. $33: 306-309$.

HAAG, H. P. \& SARRUGE, J. R. (1965) - Absorção de zinco por raízes destacadas de cafeeiro (Coffea arabica L., var. Mundo Novo). Garcia de Orta, 13 : 589-596.

HOAGLAND, D. R. \& ARNON, D. I. (1950) - The water-culture method for growing plants without soil. Berkeley, California Agrc. Exp. Sta. Circular 34\%.

JACKSON, T. L., HAY, J. \& MOORE, D. P. (1967). The effect of zinc on yield and chemical composition of sweet corn in the Willamette Valley. Amer. Soc. Hort. Sci., Proc. 91 : 462-471.

LANGIN, E. J., WARD, R. C., OLSON, R. A. \& RHOADES, H. F. (1962) - Factors responsible for poor response of corn and grain sorghum to phosphorus fertilization: III. Lime and $\mathrm{P}$ placement effects on P-Zn relations. Soil Sci. Soc. Amer. Proc. 26 : 574-578.

LARSEN, S. (1952) - The use of ${ }^{32} \mathrm{P}$ in studies on the uptake of phosphorus by plants. Plant and Soil $4: 1-10$.

LO, S. Y. \& REISENAUER, H. M. (1968) - Zinc nutrition of alfafa. Agronomy J. $60: 464-466$.

MARINHO, M. L. \& IGUE, K. (1972) - Factors affecting zinc absorption by corn from volcanic ash soils. Agronomy J. 64: 3-8.

PAULSEN, G. M. \& ROTIMI, O. A. (1968) - Phosphorus-zinc interaction in two soybean varieties differing in sensitivity to phosphorus nutrition. Soll Sci. Soc. Amer. Proc. 32 : 73-76. 
RUDGERS, L. A., DEMETRIO, J. L., PAULSEN, G. M. \& ELLIS, R. (1970). Interaction among atrazine, temperature and phosphorus-induced zinc deficiency in corn (Zea mays L.) Soil Sci. Soc. Amer. Proc. $34:$ 240-244.

RUSSELL, R. S. \& MARTIN, R. P. (1953) - A study of the absorption and utilization of phosphate by young barley plants. I. The effect of extremal concentration on the distribution of absorved phosphate between roots and shoots. Journal Exp. Bot. $4: 108-127$.

WARD, R. C., LANGIN, E. J., OLSON, R. A. \& STUKENHOLTZ, D. D. (1963) Factors responsible for poor response of corn and grain sorghum to phosphotus fertilization. III. Effects of soil compaction, moisture level and other properties on P-Zn relations. Soil Sci. Soc. Amer. Proc. $27: 326-330$.

WARNOCK, R. E. (1970) - Micronutrient uptake and mobility within corn plants (Zea mays L.) in relation to phosphorus-induced zinc deficiency. Soil Sci. Soc. Amer. Proc. 34 : 765-769. 
